\title{
Tool Use Affects Perceived Distance, But Only When You Intend to Use It
}

\author{
Jessica K. Witt, Dennis R. Proffitt, and William Epstein \\ University of Virginia
}

\begin{abstract}
Recent research demonstrates neurologic and behavioral differences in people's responses to the space that is within and beyond reach. The present studies demonstrated a perceptual difference as well. Reachability was manipulated by having participants reach with and without a tool. Across 2 conditions, in which participants either held a tool or not, targets were presented at the same distances. Perceived distances to targets within reach holding the tool were compressed compared with targets that were beyond reach without it. These results suggest that reachability serves as a metric for perception. The 3rd experiment found that reachability only influenced perceived distance when the perceiver intended to reach. These experiments suggest that the authors perceive the environment in terms of our intentions and abilities to act within it.
\end{abstract}

Keywords: egocentric distance, reaching, intention, affordances, visual perception

Near space (also called personal space, Cutting \& Vishton, 1995; and peripersonal space, Làvadas, 2002) is defined by the extent that can be reached or just slightly beyond. The present studies were designed to investigate whether the extent of reachability provides a metric for perception. Theoretical reasons to believe that it may are suggested by approaches that support a tight coupling between perception and action. The ecological approach of Gibson (1979) suggests this possibility, as does the perspective taken in the embodied cognition movement (cf. Clark, 1999). A strong version of perception/action coupling would imply that our ability to act should directly influence the way we perceive the world. To examine this possibility, we assessed whether perceived distances in near space were influenced by providing people with a hand tool that extended the distance that they could reach. We found that targets, located between the boundaries of what cannot be reached without a tool but can be reached with one, appear closer when the tool was used.

\section{Visual Sensitivities for Reachable Space: Evidence From Electrophysiology, Clinical Case Studies, and Behavior Studies of Visual Attention}

Evidence that the brain codes space in terms of reachability can be found in electrophysiology studies. Iriki, Tanaka, and Iwamura (1996) demonstrated that monkeys possess visual neurons that code for reachable space. They found neurons in the intraparietal sulcus that fired when a raisin was presented within the monkeys'

Jessica K. Witt, Dennis R. Proffitt, and William Epstein, Department of Psychology, University of Virginia.

This research was supported by National Science Foundation Information Technology Research/Carnegie Mellon Grant 0121629 and Office of Naval Research Grant N000140110060 awarded to Dennis R. Proffitt. We thank Michael Kubovy and Sarah Creem for comments on an earlier version of this article.

Correspondence concerning this article should be addressed to Jessica K. Witt, University of Virginia, P.O. Box 400400, Charlottesville, VA 22904. E-mail: jw2ex@virginia.edu arm's reach but not beyond. The monkeys were then taught to reach with a rake, which extended their reach. The "reachability" neurons adapted to this change and responded to raisins that were presented further away but within reach with the rake. This research suggests that there exist visual neurons that code for what is within reach and that these neurons adapt to changes in reachability, resulting from tool use.

Research on neglect patients demonstrates behavioral differences between what is within and beyond near space as well as the ability to remap near space. Neglect patients tend to ignore the left side of their visual field. When asked to bisect a line, they bisect only the right half, resulting in responses far to the right of true center. A double dissociation between near and far space has been shown when the bisection of lines in near space is compared with the bisection of lines in far space (Cowey, Small, \& Ellis, 1994 Halligan \& Marshall, 1991). Some patients show neglect only for near lines and not for far lines (Halligan \& Marshall), whereas other patients show neglect only for far lines (Cowey et al., 1994). For patients who showed neglect in near space only, it has been shown that reaching to far lines with a stick influences their responses to the far lines (Berti \& Frassinetti, 2000; Pegna et al., 2001). When bisecting far lines with a stick, these patients showed neglect, indicating that the space had been remapped as near space as a result of using a tool. This suggests that the behavioral differences observed between space within and beyond reach are because of the ability to reach not because of absolute distance.

Experiments in cross-modal interference also suggest different patterns of behavior to objects within and beyond reach. Participants were asked to report when and where they felt a tactile stimulus on their hand. A distracting light was presented either near or far from their hand. When the light was close, participants were less accurate and slower to report a tactile stimulus, but when it was far, accuracy was unaffected. However, when they were given a tool long enough to reach the far light, the distracting light did interfere with tactile detection (Maravita, Spence, Kennett, \& Driver, 2002). This finding and those of similar studies (e.g., Fàrne \& Làdavas, 2000; Maravita, Husain, Clarke, \& Driver, 2001) 
demonstrate that near space is remapped with tool use and that this remapping affects visual attention within this space.

The studies reviewed above show that the visual system is sensitive to the extent of reaching, either with the hand or with a held tool. Given this sensitivity, it could be the case that the extent of reachability serves as a perceptual metric in vision. That is, reaching extent could mark a perceptual discontinuity such that everything that falls within this range is perceived to be in near space, whereas everything located beyond this boundary is perceived to be outside of this immediate action space. The essence of this notion is that reachable targets are perceived as having a quality of "nearness" that targets beyond this boundary lack. Consequently, targets that cannot be reached without a tool will appear closer when a tool is held compared with when it is not.

\section{Body Scaling in Perception}

Although most of the proposed metrics in perception are defined by optical and oculomotor variables (see Proffitt \& Caudek, 2002), there are additional metrics that relate optical variables to body size. Sedgwick (1986) proposed that the perceiver's eye height provides a metric for size perception. By relating the visual angles from the top and from the bottom of an object to the eye-heightdefined horizon, one can calculate the size of the object as a proportion of the eye's elevation. Thus, eye height can provide a body-scaled metric for perceiving size. Bertamini, Yang, and Proffitt (1998) demonstrated that eye height provides a metric for relative size perception of objects viewed at a distance. They varied object height and egocentric distance, and perceivers judged which of two objects was taller. Bertamini et al. found that relative size judgments were best at eye level.

The idea that the body provides a metric for perception has roots in Gibson's theory of affordances (Gibson, 1979). Affordances were defined by Gibson as the utilities of an object given the action repertoire of the perceiver. For example, an object's form may allow a person to perceive it as graspable, tossable, rollable, or an indefinite number of other actions compatible with its size, form, substance, and weight. Experiments motivated by affordance theory have revealed, for instance, that people are sensitive to perceived sitability (Mark, 1987; Mark, Balliett, Craver, Douglas, \& Fox, 1990), climbability (Warren, 1984), and passability through gaps (Warren \& Whang, 1987). These studies show that perceptual judgments are not only determined by the properties of the environment but also influenced by the perceiver's capabilities as well. Perceived affordances relate the form and layout of objects and surfaces to the physical capabilities of the perceiver to execute actions.

\section{Perceiving the World in Terms of Action}

There is also evidence that affordances affect the perception of spatial layout. Proffitt, Bhalla, Gossweiler, and Midgett (1995) had participants estimate the slope of hills viewed from the bottom and the top. They found that these estimates were consistent from both vantage points until about $25^{\circ}$. At this slant angle, hills appear steeper from the top than from the bottom. One possible explanation for this difference in perception may be the change in the relative affordances for ascending and descending hills. It is bio- mechanically easier to maintain balance while ascending a $25^{\circ}$ hill than it is to maintain balance while descending it.

Other research has demonstrated that changes in the effort required to complete a given action affects perception of spatial layout. Participants made verbal, visual, and haptic estimates of slants from the bottom of hills (Bhalla \& Proffitt, 1999; Proffitt et al., 1995). As the effort to ascend the hill increased, by either carrying a heavy load or recovering from a long run, verbal and visual estimates of slant increased. Perceived distance also increased with effort. Objects at a distance looked farther when carrying a heavy load compared with carrying no load (Proffitt, Stefanucci, Banton, \& Epstein, 2003). Perceived distance expanded after throwing a heavy ball compared with throwing a light one (Witt, Proffitt, \& Epstein, 2004). Even though the optical information remained the same in all of these cases, changing the effort required to walk or throw influenced perceived slant or distance. These findings are consistent with the idea that we perceive the world in terms of the actions we intend to make.

\section{Overview}

In the following experiments, target distances were held constant, whereas reachability of the targets was varied. Targets were presented at varying distances within and beyond reach, and participants made verbal and visual judgments of the distance to the target. Half of the time, participants held a tool that allowed them to reach to all the targets. It was found that targets that were out of reach without the tool appeared closer when the tool was used to reach the target than when the tool was not used.

\section{Experiment 1: Verbal Estimates}

We investigated in Experiment 1 the influence of reachability on perceived distance. We manipulated reachability by having participants reach either with or without a tool. Perceived distance was measured by having participants give verbal reports of the distance to targets.

\section{Method}

Participants. Sixteen students from the University of Virginia (10 men and 6 women) participated for pay or for research credit. All gave informed consent.

Apparatus and stimuli. The participants sat in front of a rectangular table onto which stimuli were projected from a projector pointing downward from the ceiling (see Figure 1). The table top was $122 \mathrm{~cm}$ wide and $183 \mathrm{~cm}$ deep and was $97 \mathrm{~cm}$ above the floor. The table was uniformly white so as to minimize landmarks, which could influence distance judgments. A vertical wooden handle was fixed to one end of the table, and participants held this handle with their nondominant hand. The handle specified the location from which the distance to the target was to be estimated. The table was in a typically cluttered laboratory environment; however, there were no objects in the space immediately surrounding the table. During half of the experiment, participants held a $39-\mathrm{cm}$ long orchestra conductor's baton.

Procedure and design. A 2-cm white circle was projected onto the table top for $500 \mathrm{~ms}$. After it disappeared, a computer-generated voice said "touch" or "estimate." If it said "touch," and the location previously occupied by the circle was within reach, then the participant reached out and touched it; if it was beyond reach, the participant pointed to where it had been. If the computer said "estimate," the participant verbally esti- 

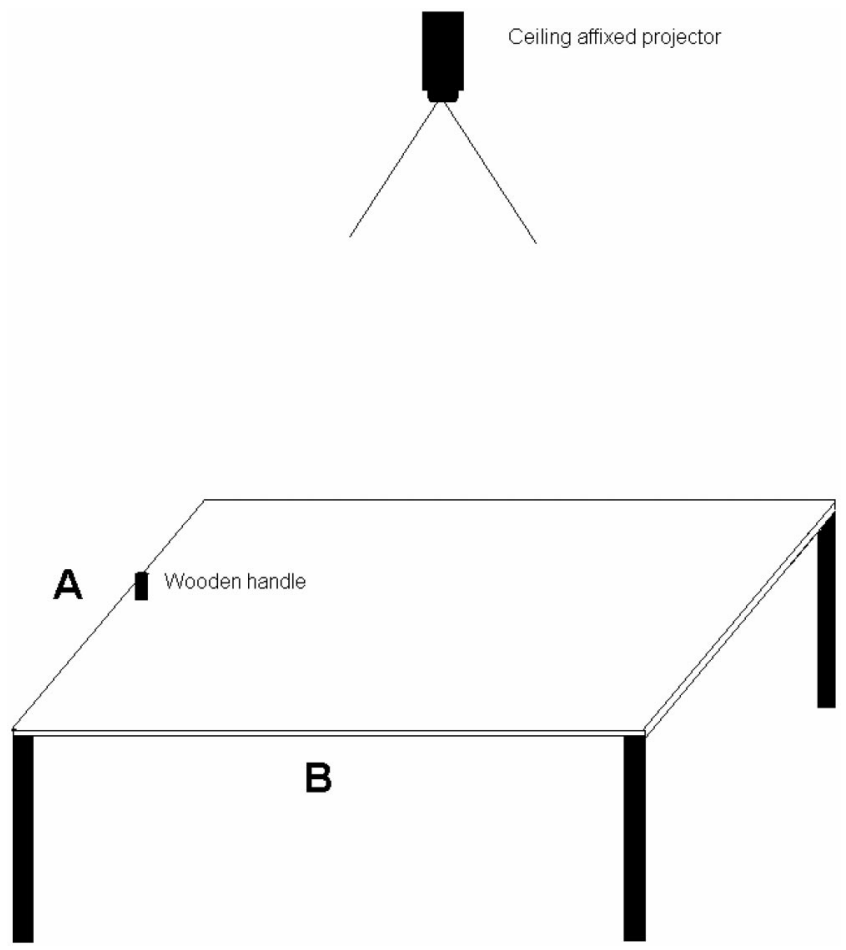

Figure 1. Set up for the experiments. Stimuli were projected from a ceiling-affixed projector onto a flat, homogeneous, white table. A wooden handle was fixed to one end. In Experiment 1, participants sat near the handle (marked by the $A$ ). In Experiments 2 and 3, participants sat at the location marked by $B$.

mated the distance in inches from the wooden handle to where the circle had been. Participants did not know whether they would have to touch or estimate the distance to each target until after the target was extinguished. Participants were not given prior training on estimating distances, and no feedback was given on reaching or estimating accuracy. Participants were not told the range of distances that would be used and were not given a source with which to calibrate their estimates.

Participants completed two blocks of trials, one with the baton and one without the baton. Each block consisted of 50 targets. Participants reached to half of the targets and estimated the distance to the other half. Reaching trials were randomly intermixed with estimating trials, so participants could not anticipate the kind of trial that would follow from the previous one. Participants were told to touch the target with the tip of their finger or with the tip of the baton. The distances used were 38.10, 40.64, 45.72, 48.26, 53.34, 54.61, 58.42, 60.96, 63.50, 71.12, 73.66, 78.74, 82.55, 91.44, $92.71,95.25,96.52,99.06,106.68$, and $109.22 \mathrm{~cm}$. Half the participants held the baton for the first block, and half held the baton for the second block. They were not aware that they would complete two blocks, so participants who held the baton in the first block did not know they would do the task again without the baton, and participants who reached with their finger in the first block did not know they would complete a second block with the baton.

Data analyses. We measured each participant's reach with and without the baton. Participants had been instructed not to lean forward; however, all participants did lean slightly forward. To take forward lean into account, we had members of our laboratory informally reach toward the targets, and we measured how far they were apt to lean. The average amount of forward lean $(13 \mathrm{~cm})$ was added to the arm lengths of the participants to account for forward lean. ${ }^{1}$ The data for analyses included the estimates for distances that were beyond arm length without the baton and within arm length with the baton. This is the area of space that is remapped into near space when reaching with the baton. The following distances were included in the analyses: 78.74, 82.55, 91.44, 92.71, 95.25, 96.52, and $99.06 \mathrm{~cm}$.

Statistical outliers were defined as values above or below 1.5 standard deviations from the mean, as determined using box plots. We excluded the data from 2 participants because $50 \%$ or more of their estimates were statistical outliers, and we excluded four individual data points that were greater than 1.5 standard deviations from the mean. These outliers are further discussed in the next section.

\section{Results and Discussion}

We ran a repeated measures analysis of variance (ANOVA), with tool and distance as independent factors and perceived distance as the dependent measure. There was a main effect for distance, $F(6,93)=3.556, p<.01, d=0.187$. There was a main effect for tool, $F(1,93)=8.599, p<.01, d=0.085$. Participants estimated the targets to be closer when they reached with a baton than when they reached or pointed with their fingers (see Figure 2). When the targets were beyond reach, they appeared to be farther away than when they were within reach. The interaction between distance and tool was not significant, $F(6,93)=0.333, p>9$.

Our results suggest that there is a difference in perceived distance when wielding a baton. The variability of the verbal estimates was quite high for all of the participants (variance $=767.23$, range $=147.32$ ) even after removing outliers (variance $=382.39$, range $=121.92$ ). Given this high variability and the necessity of removing outliers, we considered Experiment 1 to be a preliminary study. In Experiment 2, we used a measure of perceived distance that had less variability and, therefore, would be more sensitive to differences in perceived distance due to reachability.

\section{Experiment 2: Perceptual Matching}

In Experiment 2, we used the same reachability manipulation and measured perceived distance with a perceptual matching task. On a horizontal table, two comparison circles were positioned perpendicular to the line between the participants and the targets. Participants adjusted the distance between the two comparison circles to match the perceived egocentric distance to the target (see Figure 3).

\section{Method}

Participants. Eight University of Virginia students $(2$ men and 6 women) participated for pay or for research credit. All gave informed consent.

Apparatus and stimuli. Each participant sat along the long side of the same horizontal white table as in Experiment 1 (see Figure 1). This time, the table was only $36 \mathrm{~cm}$ off the ground to increase the size of the projected area. There was a small white paper circle directly in front of the participants. This circle served the same purpose as the handle in Experiment 1; the distances that were judged were the distances between this circle and the target. Participants matched the distance between the comparison circles to the distance between the paper circle and the target (see Figure 3). Participants completed half the trials holding the baton and the other half without holding the baton.

${ }^{1}$ Adding slightly different amounts of forward lean, such as $12 \mathrm{~cm}$ and $14 \mathrm{~cm}$, did not change the pattern of results. 


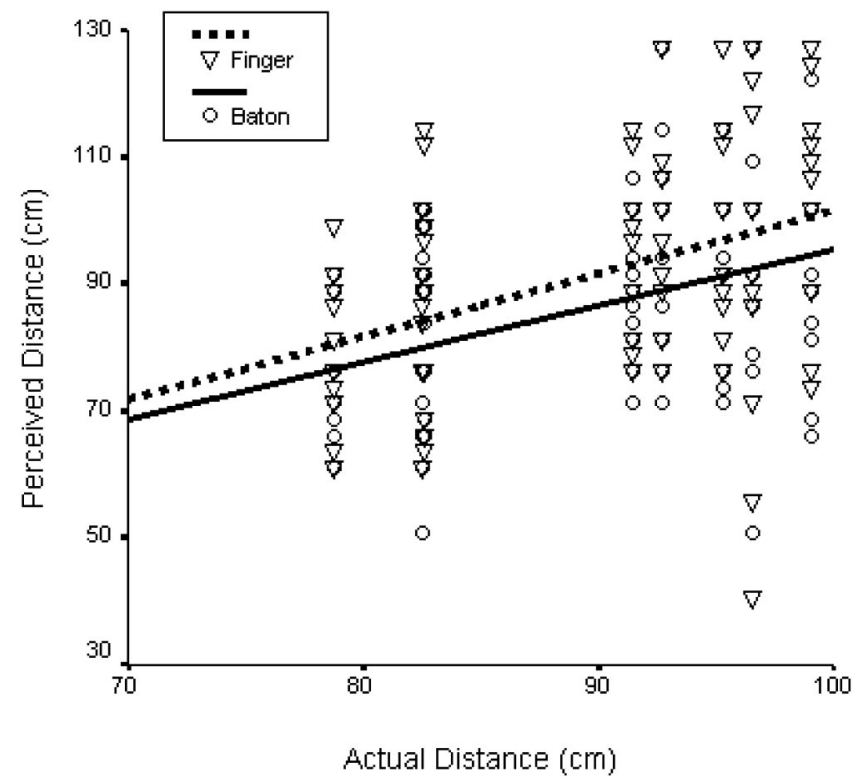

Figure 2. Verbal estimates of distance as a function of the actual distance in the finger and baton conditions of the preliminary experiment. Lines represent regression lines for each tool.

Procedure and design. On each trial, a small circle $(1 \mathrm{~cm}$ in diameter $)$ was projected beyond reach of the hand but within reach of the baton. Participants reached out and touched the circle or reached as far as they could when the circle was beyond reach. After $4 \mathrm{~s}$, two comparison circles appeared $5 \mathrm{~cm}$ on either side of the target circle (see Figure 3). Participants repositioned the comparison circles by tapping the left- and right-arrow keys on a keyboard with their nondominant hand. On every trial, participants adjusted the comparison circles closer together or farther apart until the distance between the comparison circles appeared to be the same as the distance to the target (see Figure 3). After positioning the comparison circles, participants hit the Enter key, and all the circles disappeared. Then they reached to where the target circle had been. After $4 \mathrm{~s}$, the next trial began.

Participants completed four blocks, two with the finger and two with the baton. One group of participants completed the first two blocks with their finger and then two blocks with the baton. The other group of participants completed the first two blocks with the baton and then two blocks with their finger. Each block consisted of 10 trials, with targets placed at 73.66, $78.74,83.82,88.90,93.98,99.06,104.14,109.22,114.30$, and $119.38 \mathrm{~cm}$ Participants were unaware that they were going to perform the task with both tool conditions.

\section{Results and Discussion}

Tool, distance, and order were included in a repeated measures ANOVA, with the distance between the two comparison circles as the dependent measure. There was a main effect for distance, $F(9$, $137)=31.857, p<.0001, d=0.677$. There was a main effect for tool, $F(1,137)=55.729, p<.0001, d=0.289$. Participants perceived targets to be farther away when reaching with their finger than when reaching with the baton (see Figure 4). They perceived targets that were beyond finger reach as farther than targets within reach holding the baton, even though the targets were in the same spatial location. When holding the baton, near space expanded, and targets that were remapped into near space

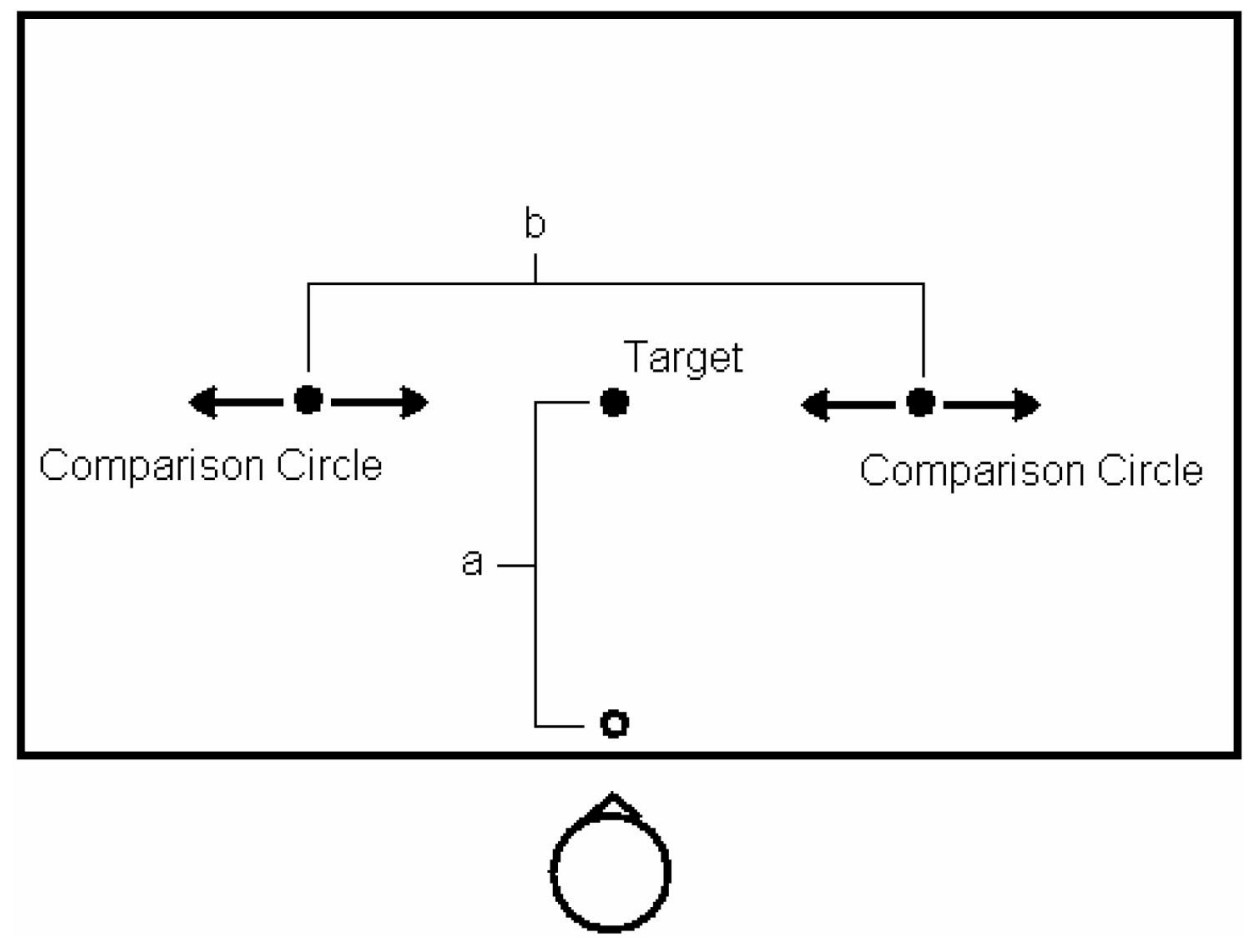

Figure 3. Perceptual matching task in Experiments 2 and 3. Participants used a keyboard to move the two comparison circles closer together or farther apart until they judged the distance between the two comparison circles (b) to be the same as the distance to the target (a). 


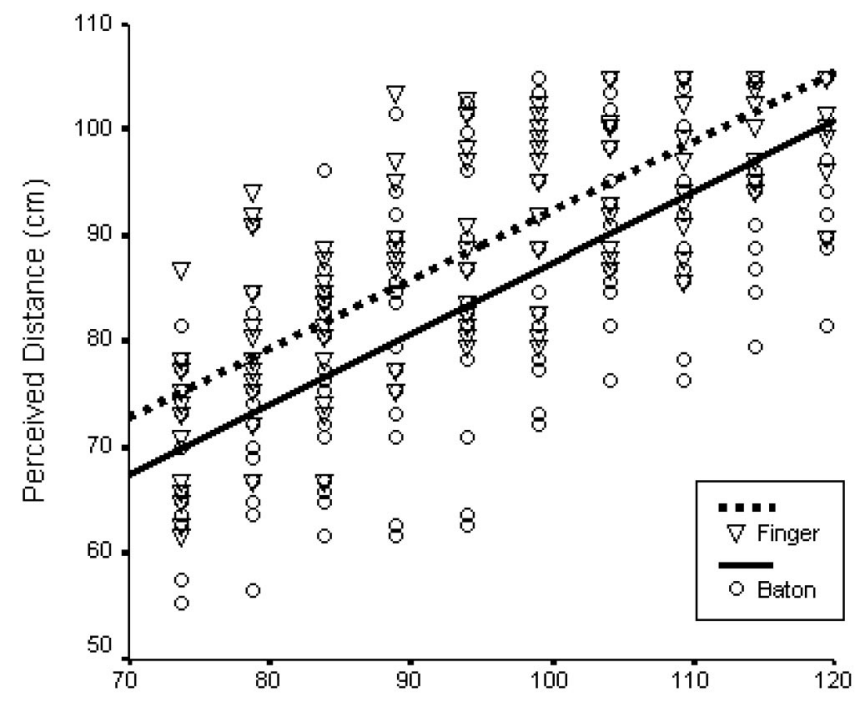

A.ctual Distance (cm)

Figure 4. Perceptually matched distance as a function of the actual distance in the finger and baton conditions of Experiment 2. Lines represent regression lines for each tool.

were perceived as being closer. Regression equations show an intercept difference of $7 \mathrm{~cm}$ between the two tool conditions (Finger: $y=.65 x+27.22$; Baton: $y=.67 x+20.70$ ), which is much less than the $39-\mathrm{cm}$ extension provided by the tool. This indicates that reachability is only one type of information contributing to perceived distance. Optical and oculomotor information also provide robust specification of apparent distance. The interaction between tool and distance was not significant, $F(9,137)=$ $0.938, p>.494$.

There was a significant effect for order, $F(1,137)=12.864$, $p<.0001, d=0.086$. The first block $(M=89.74, S D=11.28)$ looked farther than the second block $(M=85.42, S D=12.55)$. Although the interaction between session and tool was not significant, $F(1,137)=1.210, p>.72$, we ran a separate ANOVA comparing just the first block across participants. In this analysis, tool is a between-subjects variable, and because participants did not know that they were going to complete another block with the alternative tool, this analysis would reveal whether the withinsubjects effects were contaminated by cognitive correction. The tool used during the first block and actual distance were the independent measures, and perceived distance in the first block was the dependent measure. There was a main effect for tool, $F(1$, $58)=4.016, p<.05, d=0.065$, which suggests that perceived distance was influenced by the ability to reach and that our effects were not because of cognitive correction. The regression equation for finger was $y=.63 x+29.11$, and the regression equation for the baton was $y=.73 x+15.62$. There was a main effect for distance, $F(9,58)=14.555, p<.0001, d=0.693$, and the interaction between tool and distance was not significant, $F(9$, 58) $=0.264, p>.98$.

Individual participants showed different patterns of results across the two tool conditions. Regression lines for individual participants are in Figure 5. Although some participants did not show an effect of tool, this may be because of ceiling effects (see Table 1). The maximum distance that the comparison circles could be positioned was restricted by the size of the projected area, which was smaller than the width of the table. For the farther distances, many participants tried to set the comparison circles farther apart, but they were limited to this maximum distance. The participants who did show an effect of tool had fewer than three estimates that were at the limit. The participants who did not show the effect of tool had 7-15 estimates that were at the limit (see Table 1). Therefore, it is possible that these participants did not show an effect of tool because the limited space did not allow them to express differences in perceived distance. However, more work is needed to explain possible individual differences in the relationship between reachability and perceived distance.

The boundaries of the target distances were not calibrated for each participant, so some participants were able to reach to some of the targets without the baton, and others were unable to reach to some of the targets with the baton. However, when we excluded these data points, the analyses did not differ qualitatively from the analyses reported above. It is unclear whether there is a welldefined boundary of near space that produces a discontinuity in perceived distance. Perceivers' judgments of what is within reach tend to fall somewhere in between what is actually within reach if their bodies are constrained from leaning forward at the waist and what is within reach if they are allowed to bend at the waist and raise up on their toes (Rochat \& Wraga, 1997). Therefore, perceived reachability does not fall at either boundary of actual reachability. Additionally, research on neglect patients that revealed differences between near and far space did not demonstrate an abrupt change in rightward bias (Cowey, Small, \& Ellis, 1999).
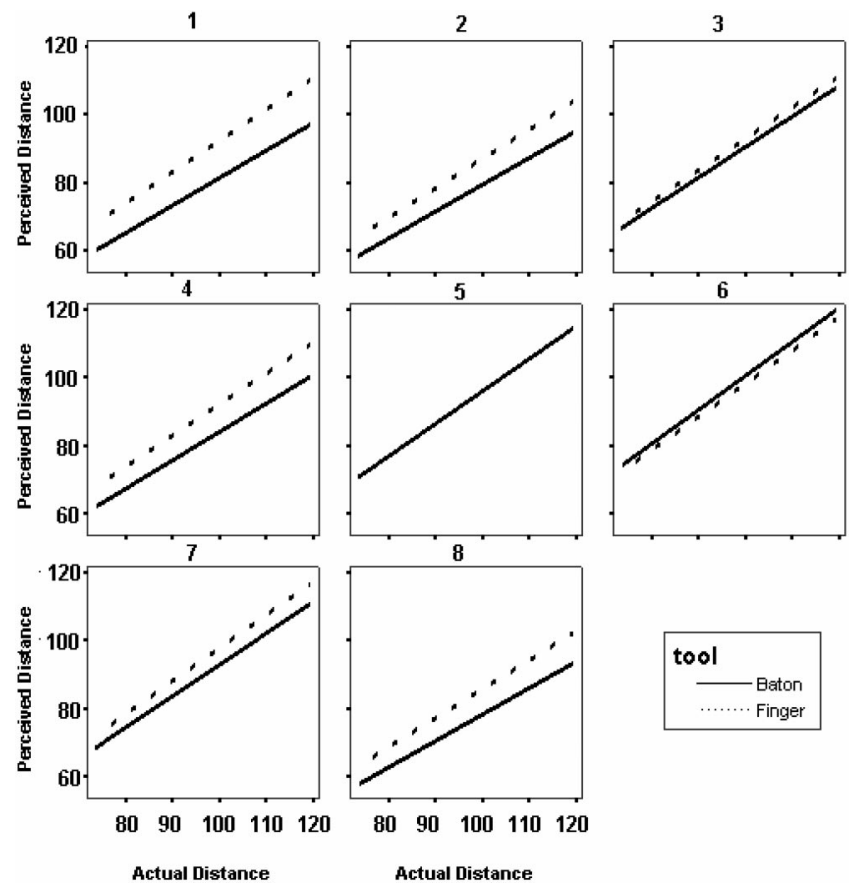

Figure 5. Perceptually matched distance as a function of the actual distance in the finger and baton conditions for individual participants in Experiment 2. Lines represent regression lines for each tool. 
Even definitions of near space do not describe near space as being a definite boundary. For example, Cutting and Vishton (1995) described near space or personal space as "generally within arm's reach and slightly beyond" (p. 100). Thus, it seems unlikely that there would be a sudden discontinuity in perceived distance.

\section{Experiment 3: Perception Without Action}

We define near space as an action space: It is reachable space. This definition implies that the perceiver must intend to reach to this space; therefore, simply holding a tool without using it to extend reach should not expand near space. In relevant studies with monkeys, visual receptive fields did not elongate when monkeys did not intend to reach (Iriki et al., 1996), and deficits in visual attention were not influenced by distractors near the end of a tool that was just being held but never manipulated (Maravita et al., 2002). In order to expand near space, the tool must be used to reach. In Experiment 3, we examined the influence of holding a tool without reaching on the perception of distance. If near space is an action space and only expands when the perceiver intends to reach, then simply holding a baton and not reaching should not expand near space. Therefore, perceived distance should not be affected by holding the baton.

\section{Method}

Participants. Eight University of Virginia students (4 men and 4 women) participated for pay or for research credit. All gave informed consent.

Apparatus and stimuli. The materials and stimuli were the same as those used in Experiment 2.

Procedure and design. The procedure and target distances were the same as those in Experiment 2, except that participants never reached to the targets. On half the trials, the participants held a baton, and on the other half of trials they did not. Participants simply watched the target circle appear without reaching to it. When the comparison circles appeared, they matched the distance between the comparison circles to the distance to the target. Then, all the circles disappeared and they waited for the next circle to appear. Participants were not told to do anything with the baton, nor were they given a reason for holding the baton.

\section{Results and Discussion}

We ran a repeated measures ANOVA, with tool and target distance as independent measures, and the distance between the

Table 1

The Tool Effects and Ceiling Counts for Individual Participants in Experiment 2

\begin{tabular}{cccc}
\hline Participant & $F(1,10)$ & $d$ & No. maximum estimates $^{\mathrm{a}}$ \\
\hline 1 & $33.45^{*}$ & 0.77 & 0 \\
2 & $15.12^{*}$ & 0.60 & 2 \\
3 & 3.08 & 0.24 & 7 \\
4 & $168.39^{*}$ & 0.96 & 12 \\
5 & 0.02 & 0.00 & 15 \\
6 & 2.48 & 0.20 & 12 \\
7 & 3.43 & 0.27 & 1 \\
8 & $36.50^{*}$ & 0.79 & \\
\hline
\end{tabular}

Note. $\quad$ No. $=$ number.

${ }^{\mathrm{a}}$ Total number of estimates $=20$.

$* p<.01$.

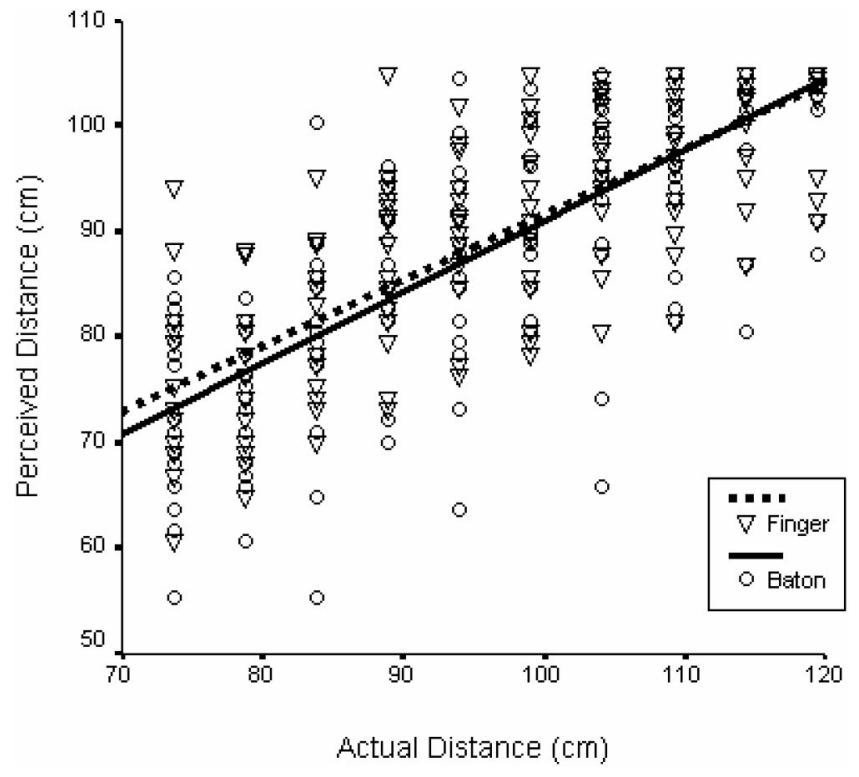

Figure 6. Perceptually matched distance as a function of the actual distance in the finger and baton conditions of Experiment 3. Lines represent regression lines for each tool.

two comparison circles as the dependent measure. Some participants did not understand the matching task at first, and these trials were excluded. There was a main effect for distance, $F(9,150)=$ 35.588, $p<.0001, d=0.68$. However, there was no effect for tool, $F(1,150)=1.814, p=.18$. Near space did not expand when participants only held the baton but did not reach with it. Consequently, perception of this space was unaffected by holding a tool (see Figure 6). ${ }^{2}$

It is not surprising that holding a tool without reaching does not influence perception because many other experiments have demonstrated that effects of tool use are conditional on intention. Reachability neurons did not fire when the monkey did not intend to reach (Iriki et al., 1996), and visual attention was not affected by holding a tool when the tool was not used to reach (Maravita et al., 2002). Our previous work also demonstrated an effect of intention: Only effort associated with an intended action affected perception (Witt et al., 2004). Effort for throwing only influenced perceived distance when the perceivers intended to throw and not when they intended to walk. Likewise, effort for walking only influenced apparent distance when the perceivers intended to walk and not when they intended to throw.

A potential concern with the present set of findings is that the results may be the result of cognitive correction. In other words, it may be the case that participants perceived the targets to be at the same distance in both tool conditions but, for reasons related to cognition as opposed to perception, reported them to be at different distances when holding the baton. The issue of cognitive correc-

\footnotetext{
${ }^{2}$ The graph suggests that there may be a difference between the two conditions for the smaller distances. However, an analysis on only the smaller distances $(70-100 \mathrm{~cm})$ and an analysis using a logarithmic scale both show that there was no difference between the two conditions ( $p>$ $.15, p>.18$, respectively).
} 
tion versus perception has been brought up with respect to perceived reachability. Heft (1993) argued that the overestimation commonly found for perceived reachability (e.g., Carello, Grosofsky, Reichel, Solomon, \& Turvey, 1989; Rochat \& Wraga, 1997) was because of an analytic attitude that influenced judgments. When participants were not given time to adjust their estimates, they were less likely to overestimate how far they could reach (Heft, 1993). However, the results from Experiment 3 suggest that the effect of tool on perceived distance is perceptual rather than analytical. If the effects in Experiments 1 and 2 were because of participants holding a baton, then they should have also adjusted their estimates when they held the baton in Experiment 3. Because the effect was only present in Experiments 1 and 2, it is probably the case that holding a baton did not induce cognitive correction.

\section{General Discussion}

Perceptual metrics derive from optical and oculomotor variables and from factors associated with the perceiver's body and abilities to act. The present experiments demonstrated that the perceiver's ability to reach to a target location is an instance of an action-based perceptual metric.

In the first two experiments, participants reached to targets with and without a baton and estimated the distance to the target. We assessed perceived distance in two ways. In the first experiment, participants verbally reported the distance to the target, and in the second experiment, participants performed a perceptual matching task. In both experiments, participants perceived the targets to be closer when they reached with a baton than when they reached with their hands. Targets within reach are perceived to be closer than targets beyond reach.

In the third experiment, we investigated whether intention mediates the influence of reachability on perceived distance. We measured perceived distance using the same perceptual matching task as in the second experiment; however, in this experiment, participants never reached. They simply did or did not hold a baton. Therefore, unlike the first two experiments, participants never intended to reach. In this latter experiment, perceived distance was found to be the same when holding the baton as when it was not held. This suggests that reachability is a metric for perceived distance only when the perceiver intends to reach. Although it seems unlikely that the effects in the first two experiments were because of differences associated with holding the baton and not because of differences in reachability, this experiment demonstrated that simply holding the baton does not influence perceived distance. Perceived distance is affected only when changes in the perceiver's ability to reach are coupled with an intention to reach.

\section{Actions, Affordances, and Perception}

Gibson was one of the first perceptual researchers to emphasize a closely coupled relationship between perception and action. Gibson (1972/2002) proposed that perceivers detect the changes in the environment that are a consequence of their movements. This relationship between perception and action develops in a reciprocal fashion, such that perceivers come to anticipate the perceptual outcomes whenever an action is planned and executed.
We have extended this notion of perception/action coupling further by suggesting that perceived spatial layout is directly influenced by intended actions. Not only do perceivers detect or anticipate changes in the environment on the basis of current or anticipated actions but also they actually see the layout of the environment in terms of their ability to act. More specifically, when perceivers anticipate the consequence of reaching to objects, they see the distance to the objects in a way that is scaled to their ability to reach.

In his later work, Gibson (1979) put forth a theory that proposed an even tighter link between action and perception: the theory of affordances. Affordances are the possibilities for action that the environment provides to a perceiver given the perceiver's action repertoire (Gibson, 1979). Thus, affordances capture the mutual fit between the environment and the perceiver. For example, only organisms having the potential to throw will perceive objects in terms of throwability; moreover, only an object that has the potential to be thrown will be perceived as having this affordance. Therefore, the affordance of throwing is only available to certain perceivers under certain environmental conditions. Furthermore, on a given occasion, perceivers will detect only a subset of the affordances that are available to them. Perceived affordances, in contrast to available affordances, depend on the perceiver's intentions. If a perceiver intends to throw, then a rock will be seen as a potential projectile; however, if the perceiver intends to break open a nut, then the rock will be seen as a nutcracker.

Perceiving affordances is finely tuned to the potential for action. Perceivers are sensitive to the boundaries at which an action is or is not possible. For example, if the height of a chair increases, then there is a specific point at which the chair does not afford sitting. Perceivers judged chairs that were within their individual boundary for sitting as being sitable and over this boundary as being not sitable (Mark, 1987). Other research has demonstrated that for each perceiver, stairs that are climbable look climbable (Mark, 1987; Warren, 1984), gaps that are crossable look crossable (Mark, Jiang, King, \& Paasche, 1999), and doorways that are passable look passable (Warren \& Whang, 1987). Furthermore, perceivers are sensitive to changes in the boundary of possible actions. Affordances are scaled to the perceiver's body, so manipulating the body affects the affordances that are available. For example, when participants wore blocks under their feet, the range of chairs that afforded sitting increased. As the participants adapted to their increased ability to sit, they judged the taller chairs to be sitable, whereas without the blocks, they judged these chairs to be not sitable (Mark, 1987).

Our present findings extend Gibson's (1979) theory of affordances in showing that perceived distance is scaled to the perceiver's intentions and abilities to act. Not only are targets perceived in terms of their reachability but also this affordance influences their apparent distances. Reachable targets are perceived as having a quality of "nearness" that targets without this affordance lack.

Although the present results are about near space and reachability, we do not believe that the effects of action potential on perception are limited to the affordance of reaching and perceived distance. There are many ways to manipulate the body, and these changes affect the affordances that are available. Many changes to the body are internalized into the perceiver's body schema (e.g., Imamizu et al., 2000; Inoue et al., 2001). We hypothesize that any change to a perceiver's body schema will influence the perception 
of the surrounding environment so long as the change is relevant for the intended action. For example, if a perceiver is wearing a cast on her or his hand and, as a result, is unable to pick up larger objects, we predict that she or he will perceive the objects to be bigger than if she or he could pick them up. Perhaps a perceiver who is carrying an umbrella and cannot pass through a doorway with the umbrella will perceive the doorway to be smaller. These effects may even extend as far as the inclusion of one's car as an extension of the body. Parking spots could look bigger when in a Porsche Boxster compared with a Lincoln Navigator. Future research is needed to delineate the generality of these effects.

\section{Possible Neural Mechanisms: Some Speculations}

The neural mechanisms that support the perception of affordances and the perception of spatial layout in terms of affordances are presently unspecified. It is likely, although not necessary, that the same neural areas are involved in both of these processes. Therefore, it is conceivable that the neurons in the intraparietal sulcus that code for reaching (Iriki et al., 1996) are responsible for driving the effect of reachability in the present experiments. Given the parallel findings between our experiments and Iriki et al.'s (1996) experiments, both of which demonstrate an effect of having a tool but only when there is an intention to use it, these reachability neurons provide a likely candidate for the neural mechanism of our effect.

If these neurons were part of the mechanism that supports our findings, then this would have implications for the two visual stream accounts of perception (Milner \& Goodale, 1995). The neurons that code for reaching (Iriki et al., 1996) are in the same area that corresponds to the dorsal stream (Ungerleider \& Mishkin, 1982), which is responsible for visually guided actions (Milner \& Goodale, 1995), and supports an unconscious perception that codes space in terms of specific actions such as reaching or grasping (e.g., Andersen, Snyder, Bradley, \& Xing, 1997; Colby \& Goldberg, 1999). These action-specific spatial codings are then used to guide specific actions. In contrast, the ventral stream, or the "what" pathway, supports conscious perception. There is evidence that information from the ventral stream can influence the dorsal stream. For example, the ventral stream informs the dorsal stream on the proper handling of objects (Creem \& Proffitt, 2001). However, there is no evidence to date suggesting that the dorsal stream informs the ventral stream about the properties of the environment. If the neurons that code for reaching in the intraparietal sulcus are responsible for the conscious perception that targets look closer when they are within reach, then this may be the first demonstration that the dorsal stream has an impact on the ventral stream and conscious perception.

\section{Summary}

At any moment in time, there are surfaces and objects surrounding us that can either be touched with our hands or are too far away. The extent of our reach defines the boundary of our immediate action space. The range of this space can be extended by having a hand tool. Perception is influenced by this affordance for immediate action. Objects that are within reach are perceived to be closer than those that are not. When a hand tool is used, objects that were previously out of reach become reachable, and, conse- quently, they appear closer than when the tool was not held. More important, this is true only when one intends to use the tool; holding a tool without anticipating its use does not influence perception. Perceived distance is a function, not only of distal extent but also of our ability and intention to act within the prescribed space.

\section{References}

Andersen, R. A., Snyder, L. H., Bradley, D. C., \& Xing, J. (1997). Multimodal representation of space in the posterior parietal cortex and its use in planning movements. Annual Review of Neuroscience, 20, 303-330.

Bertamini, M., Yang, T. L., \& Proffitt, D. R. (1998). Relative size perception at a distance is best at eye level. Perception \& Psychophysics, 60, 673-682.

Berti, A., \& Frassinetti, F. (2000). When far becomes near: Remapping of space by tool use. Journal of Cognitive Neuroscience, 12, 415-420.

Bhalla, M., \& Proffitt, D. R. (1999). Visual-motor recalibration in geographical slant perception. Journal of Experimental Psychology: Human Perception and Performance, 25, 1076-1096.

Carello, C., Grosofsky, A., Reichel, F. D., Solomon, H. Y., \& Turvey, M. T. (1989). Visually perceiving what is reachable. Ecological Psychology 1, 27-54.

Clark, A. (1999). Being there: Putting brain, body, and world together again. Cambridge, MA: MIT Press.

Colby, C. L., \& Goldberg, M. E. (1999). Space and attention in parietal cortex. Annual Review of Neuroscience, 22, 319-349.

Cowey, A., Small, M., \& Ellis, S. (1994). Left visuo-spatial neglect can be worse in far than in near space. Neuropsychologia, 32, 1059-1066.

Cowey, A., Small, M., \& Ellis, S. (1999). No abrupt change in visual hemineglect from near to far space. Neuropsychologia, 37, 1-6.

Creem, S. H., \& Proffitt, D. R. (2001). Grasping objects by their handles: A necessary interaction between cognition and action. Journal of Experimental Psychology: Human Perception and Performance, 27,218228.

Cutting, J. E., \& Vishton, P. M. (1995). Perceiving layout and knowing distances: The integration, relative potency, and contextual use of different information about depth. In W. Epstein \& S. Rogers (Eds.), Perceiving space and motion (pp. 69-117). London: Academic Press.

Fàrne, A., \& Làdavas, E. (2000). Dynamic size-change of hand peripersonal space following tool use. NeuroReport, 11, 1645-1649.

Gibson, J. J. (1979). The ecological approach to visual perception. Hillsdale, NJ: Erlbaum.

Gibson, J. J. (2002). A theory of direct visual perception. In A. T. Noe \& E. Boston (Eds.), Vision and mind: Selected readings in the philosophy of perception (pp. 77-89). Cambridge, MA: MIT Press. (Original work published 1972)

Halligan, P. W., \& Marshall, J. C. (1991, April 11). Left neglect for near but not far space in man. Nature, 350, 498-500.

Heft, H. (1993). A methodological note on overestimates of reaching distance: Distinguishing between perceptual and analytic judgments. Ecological Psychology, 5, 255-271.

Imamizu, H., Miyauchi, S., Tamada, T., Sasaki, Y., Takino, R., Putz, B., et al. (2000, January 13). Human cerebellar activity reflecting an acquired internal model of a new tool. Nature, 403, 153-154.

Inoue, K., Kawashima, R., Sugiura, M., Ogawa, A., Schormann, T., Zilles, K., \& Fukuda, H. (2001). Activation in the ipsilateral posterior parietal cortex during tool use: A PET study. NeuroImage, 14, 1469-1475.

Iriki, A., Tanaka, M., \& Iwamura, Y. (1996). Coding of modified body schema during tool use by macaque postcentral neurons. NeuroReport, 7, 2325-2330.

Làvadas, E. (2002). Functional and dynamic properties of visual peripersonal space. Trends in Cognitive Sciences, 6, 17-22. 
Maravita, A., Husain, M., Clarke, K., \& Driver, J. (2001). Reaching with a tool extends visual-tactile interactions into far space: Evidence from cross-modal extinction. Neuropsychologia, 39, 580-585.

Maravita, A., Spence, C., Kennett, S., \& Driver, J. (2002). Tool-use changes multimodal spatial interactions between vision and touch in normal humans. Cognition, 83, B25-B34.

Mark, L. S. (1987). Eyeheight-scaled information about affordances: A study of sitting and stair climbing. Journal of Experimental Psychology, 13, 361-370.

Mark, L. S., Balliett, J. A., Craver, K. D., Douglas, S. D., \& Fox, T. (1990). What an actor must do in order to perceive the affordance of sitting. Ecological Psychology, 2, 325-366.

Mark, L. S., Jiang, Y., King, S. S., \& Paasche, J. (1999). The impact of visual exploration on judgments of whether a gap is crossable. Journal of Experimental Psychology: Human Perception and Performance, 25, 287-295.

Milner, D. A., \& Goodale, M. A. (1995). The visual brain in action. Oxford, England: Oxford University Press.

Pegna, A. J., Petit, L., Caldara-Schnetzer, A.-S., Khateb, A., Annoni, J.-M., Sztajzel, R., \& Landis, T. (2001). So near yet so far: Neglect in far or near space depends on tool use. Annals of Neurology, 50, 820-822.

Proffitt, D. R., Bhalla, M., Gossweiler, R., \& Midgett, J. (1995). Perceiving geographical slant. Psychonomic Bulletin \& Review, 2, 409-428.

Proffitt, D. R., \& Caudek, C. (2002). Depth perception and perception of events. In A. F. Healy \& R. W. Proctor (Eds.), Handbook of psychology: Experimental psychology (Vol. 4, pp. 213-236). New York: Wiley.
Proffitt, D. R., Stefanucci, J., Banton, T., \& Epstein, W. (2003). The role of effort in distance perception. Psychological Science, 14, 106-112.

Rochat, P., \& Wraga, M. (1997). An account of the systematic error in judging what is reachable. Journal of Experimental Psychology: Human Perception and Performance, 23, 199-212.

Sedgwick, H. A. (1986). Space perception. In K. R. Boff, L. Kaufman, \& J. P. Thomas (Eds.), Handbook of perception and human performance Vol. 1: Sensory processes and perception (pp. 21.1-21.57). New York: Wiley.

Ungerleider, L. G., \& Mishkin, M. (1982). Two cortical visual systems. In D. J. Ingle, M. A. Goodale, \& R. J. W. Mansfield (Eds.), Analysis of visual behavior (pp. 549-586). Cambridge, MA: MIT Press.

Warren, W. H. (1984). Perceiving affordances: Visual guidance of stair climbing. Journal of Experimental Psychology: Human Perception and Performance, 10, 683-703.

Warren, W. H., \& Whang, S. (1987). Visual guidance of walking through apertures: Body-scaled information for affordances. Journal of Experimental Psychology: Human Perception and Performance, 13, 371-383.

Witt, J. K., Proffitt, D. R., \& Epstein, W. (2004). Perceiving distance: A role of effort and intent. Perception, 33, 577-590.

Received March 22, 2004

Revision received February 3, 2005

Accepted February 11, 2005

\section{New Editors Appointed, 2007-2012}

The Publications and Communications (P\&C) Board of the American Psychological Association announces the appointment of three new editors for 6-year terms beginning in 2007. As of January 1, 2006, manuscripts should be directed as follows:

- Journal of Experimental Psychology: Learning, Memory, and Cognition (www.apa.org/journals/ xlm.html), Randi C. Martin, PhD, Department of Psychology, MS-25, Rice University, P.O. Box 1892, Houston, TX 77251.

- Professional Psychology: Research and Practice (www.apa.org/journals/pro.html), Michael C. Roberts, PhD, 2009 Dole Human Development Center, Clinical Child Psychology Program, Department of Applied Behavioral Science, Department of Psychology, 1000 Sunnyside Avenue, The University of Kansas, Lawrence, KS 66045.

- Psychology, Public Policy, and Law (www.apa.org/journals/law.html), Steven Penrod, PhD, John Jay College of Criminal Justice, 445 West 59th Street N2131, New York, NY 10019-1199.

Electronic manuscript submission. As of January 1, 2006, manuscripts should be submitted electronically through the journal's Manuscript Submission Portal (see the Web site listed above with each journal title).

Manuscript submission patterns make the precise date of completion of the 2006 volumes uncertain. Current editors, Michael E. J. Masson, PhD, Mary Beth Kenkel, PhD, and Jane GoodmanDelahunty, $\mathrm{PhD}$, JD, respectively, will receive and consider manuscripts through December 31, 2005. Should 2006 volumes be completed before that date, manuscripts will be redirected to the new editors for consideration in 2007 volumes.

In addition, the P\&C Board announces the appointment of Thomas E. Joiner, PhD (Department of Psychology, Florida State University, One University Way, Tallahassee, FL 32306-1270), as editor of the Clinician's Research Digest newsletter for 2007-2012. 